\title{
On some new characterizations of weakly compact sets in Banach spaces
}

\author{
by \\ LiXin Cheng, Qinguin Cheng and Zhenghua Luo (Xiamen)
}

\begin{abstract}
We show several characterizations of weakly compact sets in Banach spaces. Given a bounded closed convex set $C$ of a Banach space $X$, the following statements are equivalent: (i) $C$ is weakly compact; (ii) $C$ can be affinely uniformly embedded into a reflexive Banach space; (iii) there exists an equivalent norm on $X$ which has the $w 2 R$-property on $C$; (iv) there is a continuous and $w^{*}$-lower semicontinuous seminorm $p$ on the dual $X^{*}$ with $p \geq \sup _{C}$ such that $p^{2}$ is everywhere Fréchet differentiable in $X^{*}$; and as a consequence, the space $X$ is a weakly compactly generated space if and only if there exists a continuous and $w^{*}$-l.s.c. Fréchet smooth (not necessarily equivalent) norm on $X^{*}$.
\end{abstract}

1. Introduction. Because of the importance of weak compactness in both Banach space theory and its applications, the study of properties of weakly compact sets has been continued for over 80 years. A large number of achievements, such as the Choquet integral representation theorem, the Eberlein-Smulian theorem, James' characterization of weakly compact sets [J], the Davis-Figiel-Johnson-Pełczyński theorem [DFJP], the OrliczPettis theorem, the Krein-Milman theorem, the Krein-Smulian theorem and Rainwater's theorem (see, for instance, [D2], [F], [L] and the references therein), have been obtained in both the theory and applications.

The study of topological and renorming properties of their relativesreflexive Banach spaces and weakly compactly generated (WCG) spaces, has also attracted attention of many mathematicians. Amir and Lindenstrauss AL first introduced the notion of weakly compactly generated Banach space and proved that for every WCG space $X$ there is a continuous one-to-one mapping $T: X \rightarrow c_{0}(\Gamma)$ for some set $\Gamma$. Troyanski's renorming theorem [T] says that every WCG space admits an equivalent locally uniformly ro-

2010 Mathematics Subject Classification: Primary 46A55, 46B20, 46B50; Secondary 46B03, 58C20, 46G05.

Key words and phrases: weakly compact set, renorming, smoothness, convexity, WCG space, Banach space. 
tund (LUR) norm. This theorem, combined with Asplund's averaging technique [As], implies that every reflexive space has an equivalent norm such that both the norm and its dual are LUR (hence, both are Fréchet smooth). We should mention that Fabian, Godefroy, Montesinos and Zizler [FGMZ] showed a number of fine characterizations of WCG spaces and their subspaces. Fabian, Montesinos and Zizler further proved some new characterizations of weakly compact sets [FMZ1] and new renorming characterizations of subspaces of WCG spaces in terms of $\varepsilon$-M smooth norms, $\varepsilon$-M LUR norms and $\sigma$-Asplund generated spaces [FMZ2].

Let us mention that a recent renorming theorem of Odell and Schlumprecht $[\mathrm{OS}]$ presents a new renorming characterization of reflexive spaces: a sufficient and necessary condition for a separable Banach space $X$ to be reflexive is that $X$ admits an equivalent norm with the $2 R$-property, i.e., for any bounded sequence $\left(x_{n}\right) \subset X$, the condition

$$
\lim _{m} \lim _{n}\left\|x_{m}+x_{n}\right\|=\lim _{n} 2\left\|x_{n}\right\|
$$

implies that $\left(x_{n}\right)$ converges. More recently, Hájek and Johanis [HJ] gave a similar renorming characterization of general reflexive Banach spaces: a Banach space $X$ is reflexive if and only if it admits an equivalent norm with the $w 2 R$-property, i.e., if a bounded sequence $\left(x_{n}\right) \subset X$ satisfies (1), then $\left(x_{n}\right)$ converges weakly.

The aim of this paper is to present some new characterizations of weakly compact sets in Banach spaces. Our main results are the following.

TheOREM 1.1. For a nonempty bounded closed convex set $C$ of a Banach space $X$, the following statements are equivalent:

(i) $C$ is weakly compact;

(ii) $C$ can be affinely uniformly embedded into a reflexive Banach space;

(iii) there exists an equivalent norm on $X$ which has the w2R-property on $C$ (2R-property on $C$, if $C$ is separable);

(iv) there is a continuous and $w^{*}$-lower semicontinuous (l.s.c.) seminorm $p$ on the dual $X^{*}$ with $p \geq \sup _{C}$ such that $p^{2}$ is everywhere Fréchet differentiable in $X^{*}$.

As a consequence of (iv) above, we have

COROLlary 1.2. The space $X$ is a weakly compactly generated space if and only if there exists a continuous and $w^{*}$-l.s.c. Fréchet smooth (not necessarily equivalent) norm on $X^{*}$.

The letter $X$ will always denote a Banach space and $X^{*}$ its dual. For a subset $A \subset X$, we denote by $\bar{A}$, co $A, \sigma_{A} \equiv \sup _{A}(\cdot)$ and $A^{0} \equiv\left\{x^{*} \in X^{*}\right.$ : $\left.\left\langle x^{*}, x\right\rangle \leq 1, \forall x \in A\right\}$ the closure of $A$, the convex hull of $A$, the support function of $A$ on $X^{*}$ and the polar of $A$, respectively. 
2. A characterization of weakly compact sets by affine uniform embedding. In this section we show an embedding condition for a bounded closed convex set in a Banach space $X$ to be weakly compact, or to generate a reflexive subspace of $X$. Our main tools are the Davis-Figiel-JohnsonPełczyński lemma [DFJP and James' characterization of weakly compact sets [J].

For a Banach space $X$, we write dens $X$ for the density of $X$, that is, the minimal cardinality of a dense set in $X$ (see, for instance, [DGZ, p. 58]). The following factorization theorem is due to Davis-Figiel-Johnson-Pełczyński [DFJP] (see also [D1]).

Theorem 2.1. Suppose that $(X,\|\cdot\|)$ is a Banach space with the closed unit ball $B$. Let $K$ be a convex symmetric bounded subset of $X$. For each positive integer $n$, let $U_{n}=2^{n} K+2^{-n} B$. Denote by $\|\cdot\|_{n}$ the Minkowski functional generated by $U_{n}$, i.e.

$$
\|x\|_{n}=\inf \left\{t>0: x \in t U_{n}\right\} .
$$

For $x \in X$, let $|x|=\left(\sum_{n=1}^{\infty}\|x\|_{n}^{2}\right)^{1 / 2}$ and $Y=\{x \in X:|x|<\infty\}$. Denote by $C$ the closed unit ball of $(Y,|\cdot|)$. Let $j: Y \rightarrow X$ be the natural inclusion mapping. Then

(i) $K \subset C$;

(ii) $(Y,|\cdot|)$ is a Banach space and $j$ is continuous;

(iii) $j^{* *}: Y^{* *} \rightarrow X^{* *}$ is one-to-one and $Y=j^{* *-1}(X)$;

(iv) $(Y,|\cdot|)$ is reflexive if and only if $K$ is relatively weakly compact in $X$.

The following is a consequence of the DFJP factorization theorem above.

THEOREM 2.2. Under the assumptions of Theorem $2.1, j^{-1}$ is uniformly continuous on $K$ and $\operatorname{dens}(Y,|\cdot|)=\operatorname{dens}(Y,\|\cdot\|)$ (hence, $(Y,|\cdot|)$ is separable if and only if $(Y,\|\cdot\|)$ is $)$.

A result similar to Theorem 2.2 is presented in [CCLZ]. Here, for the sake of convenience, we give a simple proof.

Proof of Theorem 2.2. For every $m \in \mathbb{N}$, let

$$
P_{m}(x)=\left(\sum_{n=1}^{m}\|x\|_{n}^{2}\right)^{1 / 2} \text {. }
$$

Then $P_{m}$ is uniformly $\|\cdot\|$-continuous, since every $\|\cdot\|_{n}$ is uniformly $\|\cdot\|$ continuous on $X$. Note that $\|x\|_{n}<2^{-n}$ for every $n \in \mathbb{N}$ and $x \in K$. Therefore, $P_{m}$ uniformly converges to $|\cdot|$ on $K$, which implies $|\cdot|$ is uniformly $\|\cdot\|$-continuous on $K$.

Since dens $(Y,\|\cdot\|)=\operatorname{dens}\left(Y,\|\cdot\|_{n}\right)$ for all $n \in \mathbb{N}$, the density of the direct $\operatorname{sum} \sum \oplus\left(Y,\|\cdot\|_{n}\right)$ equipped with the norm $\left|\left(x_{n}\right)\right|=\left(\sum_{n=1}^{\infty}\left\|x_{n}\right\|_{n}^{2}\right)^{1 / 2}$, i.e., $\operatorname{dens}(Y,|\cdot|)$, must be equal to $\operatorname{dens}(Y,\|\cdot\|)$. 
Proposition 2.3. Suppose that $C$ is a closed convex set in a Banach space $X$. Then the following statements are equivalent:

(i) $C$ is weakly compact;

(ii) for every sequence $\left(x_{n}\right) \subset C$, there is a convergent sequence $\left(y_{n}\right)$ satisfying $y_{n} \in \operatorname{co}\left\{x_{j}\right\}_{j \geq n}$ for all $n \in \mathbb{N}$;

(iii) for every sequence $\left(x_{n}\right) \subset C$, there is a weakly convergent sequence $\left(y_{n}\right)$ satisfying $y_{n} \in \operatorname{co}\left\{x_{j}\right\}_{j \geq n}$ for all $n \in \mathbb{N}$.

Proof. (i) $\Rightarrow$ (ii) is a classical result.

(ii) $\Rightarrow$ (iii) is trivial.

(iii) $\Rightarrow$ (i). We will use James' theorem [J] to obtain the conclusion by showing that every $x^{*} \in X^{*}$ attains its maximum on $C$.

Given $x^{*} \in X^{*}$, let $x_{n} \in C$ be such that $\left\langle x^{*}, x_{n}\right\rangle \geq \sigma_{C}\left(x^{*}\right)-1 / n$ for all $n \in \mathbb{N}$. By (iii), there is a sequence $\left(y_{n}\right)$, with $y_{n} \rightarrow y_{0} \in C$ in the weak topology, satisfying $y_{n} \in \operatorname{co}\left\{x_{j}\right\}_{j \geq n}$ for all $n \in \mathbb{N}$. Clearly, $\left\langle x^{*}, y_{n}\right\rangle \geq$ $\sigma_{C}\left(x^{*}\right)-1 / n$ for all $n \in \mathbb{N}$ and $\left\langle x^{*}, y_{0}\right\rangle=\sigma_{C}\left(x^{*}\right)$.

It is well known that a bounded linear operator between two normed spaces is norm-to-norm continuous if and only if it is weak-to-weak continuous (see, for instance, [D2, p. 12]). The following lemma is a localized version of this result.

Lemma 2.4. Suppose that $X, Y$ are Banach spaces and $C \subset X$ and $D \subset Y$ are closed convex sets. Let $\rho_{X}$ be the metric induced by the norm of $X$. Suppose that $T:\left(C, \rho_{X}\right) \rightarrow\left(D, \rho_{Y}\right)$ is a continuous affine mapping. If $C$ is weakly compact in $X$, then $T C$ is weakly compact in $Y$.

Proof. By James' theorem again, every functional $y^{*} \in Y$ attains its maximum on $T C$, under the hypothesis that $C$ is weakly compact. Since $C$ is bounded convex and since $T$ is a continuous affine mapping, $T C$ is necessarily bounded and convex. Let $y^{*} \in Y^{*}$, and let $\left(y_{n}\right) \subset T C$ be such that $\sigma_{T C}\left(y^{*}\right)-1 / n \leq\left\langle y^{*}, y_{n}\right\rangle$ for all $n \in \mathbb{N}$. Next, let $x_{n} \in C$ be such that $y_{n}=$ $T x_{n}$ for $n=1,2, \ldots$ By Theorem 2.3, the convexity and weak compactness of $C$ imply that there exists a sequence $\left(z_{n}\right) \subset C$ with $z_{n} \in \operatorname{co}\left\{x_{j}\right\}_{j \geq n}$ for all $n \in \mathbb{N}$, and $z \in C$ such that $z_{n} \rightarrow z$. The continuity and affinity of $T$ yield $T z_{n} \rightarrow T z \in T C$. Note that $T z_{n} \in T \operatorname{co}\left\{x_{j}\right\}_{j \geq n}=\operatorname{co}\left\{T x_{j}\right\}_{j \geq n}$. We observe that $\left\langle y^{*}, T z_{n}\right\rangle \rightarrow\left\langle y^{*}, T z\right\rangle=\sigma_{T C}\left(y^{*}\right)$.

Let $\left(U, d_{U}\right),\left(V, d_{V}\right)$ be two metric spaces and $f: U \rightarrow V$ be a mapping. We denote by $\omega_{f}$ the modulus of continuity of $f$ defined as follows:

$$
\omega_{f}(t)=\sup \left\{d_{V}(f(x), f(y)): x, y \in U, d_{U}(x, y) \leq t\right\}, \quad \forall t>0,
$$

and by $\operatorname{Lip}(f)$ the Lipschitz norm of $f$ :

$$
\operatorname{Lip}(f)=\sup \left\{\frac{d_{V}(f(x), f(y))}{d_{U}(x, y)}: x, y \in U, d_{X}(x, y) \neq 0\right\} .
$$


If $\lim _{t \rightarrow 0} \omega_{f}(t)=0$, then $f$ is called uniformly continuous; if $\operatorname{Lip}(f)<\infty$, then $f$ is called a Lipschitz mapping.

DeFinition 2.5. With $U, V$ and $f$ as above,

(i) $f$ is a uniform embedding from $U$ to $V$ if $f$ is injective and both $f$ and $f^{-1}$ are uniformly continuous;

(ii) $f$ is a Lipschitz embedding from $U$ to $V$ if $f$ is injective and both $f$ and $f^{-1}$ are Lipschitz.

TheOREM 2.6. Suppose that $C$ is a nonempty bounded closed convex subset of a Banach space $X$. Then

(i) $C$ can be affinely uniformly embedded into a reflexive space $Y$ if and only if $C$ is weakly compact in $X$;

(ii) $C$ can be Lipschitz embedded into a reflexive space $Y$ if and only if the closure of $\operatorname{span} C$ is itself a reflexive subspace of $X$.

Proof. (i) The sufficiency is a direct consequence of (ii) and (iii) of Theorem 2.1 .

The necessity follows immediately from Lemma 2.4.

(ii) It suffices to show the necessity. Assume that $Y$ is a reflexive space and $f: C \rightarrow Y$ is a Lipschitz embedding. Let $X_{C}=\overline{\operatorname{span}} C$. To show that $X_{C}$ is reflexive, it suffices to prove that each separable closed subspace of $X_{C}$ is reflexive. Let $X_{0} \subset X_{C}$ be a closed separable subspace. The density of span $C$ in $X_{C}$ implies that there is a sequence $\left(x_{n}\right) \subset C$ such that $X_{0} \subset X_{1} \equiv$ $\overline{\operatorname{span}}\left\{x_{n}\right\}$. Let $C_{1}=\overline{\mathrm{co}}\left\{x_{n}\right\}$. Clearly, $C_{1}$ is closed, convex and fundamental in $X_{1}$. Now, we consider the Lipschitz embedding $f_{1}: C_{1} \rightarrow Y$, the restriction of $f$ to $C_{1}$. Note that $Y$ is reflexive (hence, it has the Radon-Nikodým property). By a generalized Gâteaux differentiability theorem [CZ], there exist a point $x_{0} \in C_{1}$ and a bounded linear operator $T: X_{1} \rightarrow Y$ such that for all $x \in X_{1}$,

$$
\lim _{t \rightarrow 0^{+}} \frac{f\left(x_{0}+t x\right)-f\left(x_{0}\right)}{t}=T x .
$$

It is easy to see that $T: X_{1} \rightarrow Y$ is a linear embedding. The reflexivity of $Y$ implies that $X_{1}$ is itself reflexive. Therefore, $X_{0}$ is reflexive.

\section{A feature of weakly compact sets via Fréchet smooth renorm-}

ing. In this section, we will discuss Fréchet smooth renorming property on the dual $X^{*}$ with respect to a weakly compact set of a Banach space $X$. First, we need some preparation.

For a convex function $f$ defined on a Banach space $X$, its subdifferential mapping $\partial f: X \rightarrow 2^{X^{*}}$ is defined by

$$
\partial f(x)=\left\{x^{*} \in X^{*}: f(x+y)-f(x) \geq\left\langle x^{*}, y\right\rangle, \forall y \in X\right\},
$$


and its conjugate function $f^{*}$ by

$$
f^{*}\left(x^{*}\right)=\sup \left\{\left\langle x^{*}, x\right\rangle-f(x): x \in X\right\}, \quad x^{*} \in X^{*} .
$$

We say that the convex function $f$ is Gâteaux (resp. Fréchet) differentiable at $x$ if there is $x^{*} \in X^{*}$ such that

$$
\lim _{t \rightarrow 0^{+}} \frac{f(x+t y)-f(x)}{t}-\left\langle x^{*}, y\right\rangle=0, \quad \forall y \in X,
$$

respectively,

$$
\lim _{t \rightarrow 0^{+}} \sup _{y \in B_{X}}\left[\frac{f(x+t y)-f(x)}{t}-\left\langle x^{*}, y\right\rangle\right]=0 .
$$

In the latter case, we denote $x^{*}=d f(x)$.

Suppose that $C^{*} \subset X^{*}$ is a bounded $w^{*}$-closed convex set in the dual $X^{*}$ of $X$. A point $x^{*} \in C^{*}$ is said to be a $w^{*}$-strongly exposed point of $C^{*}$ if there is $x \in X$ such that for any sequence $x_{n}^{*} \subset C^{*},\left\langle x_{n}^{*}, x\right\rangle \rightarrow\left\langle x^{*}, x\right\rangle$ implies $x_{n}^{*} \rightarrow x^{*}$. In this case, $x^{*}$ is said to be strongly exposed by $x$.

Proposition 3.1 ([ㅍ, Prop. 5.11]). Let $p$ be a continuous Minkowski functional on $X$ and let $C^{*}=\left\{x^{*} \in X^{*}: x^{*} \leq p\right\}$. Then $p$ is Fréchet differentiable at $x$ with $d p(x)=x^{*} \in C^{*}$ if and only if $x^{*}$ is a $w^{*}$-strongly exposed point of $C^{*}$ and it is strongly exposed by $x$.

Proposition 3.2 ([P, Lemma 5.10]). Let $p$ be a continuous Minkowski functional on $X$ and let $C^{*}=\left\{x^{*} \in X^{*}: x^{*} \leq p\right\}$. Then $x^{*} \in \partial p(x)$ if and only if $x^{*} \in C^{*}$ with $\left\langle x^{*}, x\right\rangle=p(x)$.

Proposition 3.3 ([WC1], see also [CW]). Suppose that $X$ is a Banach space and $C \subset X$ is a bounded closed set. Denote by $C^{* *}$ the $w^{*}$-closed convex hull of $C \subset X^{* *}$. Then every $w^{*}$-strongly exposed point of $C^{* *}$ is in $C$.

Proposition 3.4 ([CLS, Prop. 2.2]). Let $f$ be a continuous convex function on $X$ with $f(0)=-1$. Let $p$ be the Minkowski functional generated by epi $f$, the epigraph of $f$. Then $f$ is Fréchet differentiable at $x$ with Fréchet derivative $d f(x)=x^{*}$ if and only if $p$ is Fréchet differentiable at $z \equiv(x, f(x))$ with Fréchet derivative $d p(z)=\left(y^{*}, r\right)$, where $y^{*}=x^{*} / f^{*}\left(x^{*}\right)$ and $r=$ $-1 / f^{*}\left(x^{*}\right)$.

LEMMA 3.5. Suppose that $f$ is a continuous and $w^{*}$-l.s.c. convex function defined on the dual $X^{*}$ of $X$.

(i) If $f$ is Fréchet differentiable at $x^{*}$ with Fréchet derivative $d f\left(x^{*}\right)=$ $x^{* *}$, then $x^{* *} \in X$.

(ii) If, in addition, $f$ is sublinear, then $x^{* *} \in C \equiv C^{* *} \cap X$, where $C^{* *}=$ $\left\{x^{* *} \in X^{* *}: x^{*} \leq f\right\}$.

Proof. We first show (ii). Without loss of generality, we can assume $f \geq 0$. Therefore, $f$ is a continuous and $w^{*}$-l.s.c. Minkowski functional on $X^{*}$. 
Let $d f\left(x^{*}\right)=x^{* *}$. By Proposition 3.1, $x^{* *}$ is a $w^{*}$-strongly exposed point of $C^{* *}$, and it is strongly exposed by $x^{*}$. Since $f$ is $w^{*}$-l.s.c., $C^{* *}$ is just the $w^{*}$-closure of $C$. Proposition 3.3 yields $x^{* *} \in C$.

(i) We can assume that $x^{*}=0$ with $f(0)=-1$. Let $p$ be the Minkowski functional generated by epi $f$. Then $p$ is continuous and $w^{*}$-l.s.c. Since $f$ is Fréchet differentiable at 0 , by Proposition 3.4, we see that $p$ is also Fréchet differentiable at $(0,-1)$ and with $d p(0,-1)=(d f(0),-1)$. By (ii) just proven, $(d f(0),-1) \in X \times \mathbb{R}$, i.e., $d f(0) \in X$.

TheOREM 3.6. Suppose that $K$ is a nonempty bounded subset of a $B a$ nach space $(X,\|\cdot\|)$. Then $K$ is relatively weakly compact if and only if $X^{*}$ admits a $w^{*}$-l.s.c. seminorm $q$ such that

(i) $q^{2}$ is Fréchet differentiable everywhere in $X^{*}$;

(ii) for some $\lambda>0, \lambda\|\cdot\| \geq q \geq \sigma_{K}$ on $X^{*}$.

Proof. Since $K$ is relatively weakly compact if and only if $\overline{c o}\{K \cup-K\}$ is weakly compact, we can assume, without loss of generality, that $K$ is itself absolutely convex closed.

Sufficiency. Suppose that $q$ is a continuous and $w^{*}$-l.s.c. seminorm on $X^{*}$ with $\lambda\|\cdot\| \geq q \geq \sigma_{K}$, for some $\lambda>0$, such that $q^{2}$ is Fréchet differentiable on $X^{*}$. We can assume that $\lambda=1$, and that $q$ is actually a norm on $X^{*}$. Indeed, otherwise let $Y=\left\{x^{*} \in X^{*}: q\left(x^{*}\right)=0\right\}$. Then $Y$ is a $w^{*}$-closed subspace of $X^{*}$, and we turn to the quotient space $Y^{0^{*}}=X^{*} / Y$ and the Fréchet smooth quotient norm $\widetilde{q}$ defined by $\widetilde{q}\left(x^{*}+Y\right)=q\left(x^{*}\right)$ for $x^{*} \in X^{*}$.

Let $C^{* *}=\left\{x^{* *} \in X^{* *}: x^{* *} \leq q\right\}$. Then $C^{* *}$ is a bounded $w^{*}$-closed absolutely convex set, $q\left(x^{*}\right)=\sigma_{C^{* *}}\left(x^{*}\right)=\sigma_{C}\left(x^{*}\right)$ for all $x^{*} \in X^{*}$, and $K \subset C \equiv C^{* *} \cap X$. Note that $C^{* *}$ is the $w^{*}$-closure of $C$, and $q \geq \sigma_{K}$ implies $K \subset C$. It suffices to show that $C$ is weakly compact. Thanks again to James' characterization of weakly compact sets [J], we need only show that every functional in $X^{*}$ attains its maximum on $C$. It is enough to show this for every $x^{*} \in X^{*}$ with $q\left(x^{*}\right)=1$, since $S_{\left(X^{*}, q\right)} \cup\{0\}$ is an absorbing set of $X^{*}$. Given such an $x^{*}$, by Lemma 3.4 and Proposition 3.2, $x \equiv d q\left(x^{*}\right) \in C$ and $\left\langle x^{*}, x\right\rangle=q\left(x^{*}\right)=1$.

Necessity. Let $X_{0}$ be the closure of $\operatorname{span} K$ in $X$. Since $K$ is also weakly compact in $X_{0}$, by Theorem 2.1 there is a reflexive space $(Y,|\cdot|)$ such that $K \subset C \equiv B_{Y} \subset B_{X_{0}}$. The reflexivity of $Y$ allows us to assume that the dual norm $|\cdot|^{*}$ is both locally uniformly convex and Fréchet smooth on $Y^{*}$. Therefore, within the natural norm-preserving restriction to $Y$, we obtain $X_{0}^{*} \subset Y^{*}$ and $B_{X_{0}^{*}} \subset B_{Y^{*}}$. These further imply that $|\cdot|^{*}$ is a Fréchet smooth and $w^{*}$-l.s.c. norm on $X_{0}^{*}=X^{*} / X_{0}^{0}$. Now, we define $q$ on $X^{*}$ by $q\left(x^{*}\right)=\left|Q\left(x^{*}\right)\right|^{*}$ for $x^{*} \in X^{*}$, where $Q: X^{*} \rightarrow X^{*} / X_{0}^{0}$ denotes the quotient mapping. Then it is easy to see that $q$ satisfies (i) and (ii) of the theorem. 
Corollary 3.7. A Banach space $X$ is a WCG space if and only if there is a continuous and $w^{*}$-l.s.c. norm (not necessarily equivalent) on $X^{*}$ that is everywhere Fréchet differentiable off the origin.

Proof. Since $X$ is WCG if and only if there is a weakly compact and absolutely convex subset $K$ of $X$ such that $X=\overline{\operatorname{span}} K$, by Theorem 3.6 it suffices to note that $\sigma_{K}$ is a norm on $X^{*}$.

\section{A characterization of weakly compact sets through renorm-} ing with the $2 \mathrm{R}$-property. In this section we extend or localize the recent renorming theorems of Odell-Schlumprecht OS and Hájek-Johanis [HJ]. Consequently, a new characterization of weakly compact convex sets is obtained.

Definition $4.1([\mathrm{HJ}])$. We say that a closed convex set $C$ of a Banach space $X$ has the $2 R$ ( $w 2 R$, resp.)-property if for every bounded sequence $\left(x_{n}\right) \subset C$, if $\lim _{m} \lim _{n}\left[2\left(\left|x_{m}\right|^{2}+\left|x_{n}\right|^{2}\right)-\left|x_{m}+x_{n}\right|^{2}\right]=0$, then $\left(x_{n}\right)$ converges (weakly, resp.) In this case, we also say that the norm has the $2 R(w 2 R$, resp.)-property on $C$.

REMARK 4.2. By the definition we can easily observe that a norm $|\cdot|$ of $X$ has the $2 R(w 2 R)$-property on a closed convex set $C$ if and only if for every bounded sequence $\left(x_{n}\right) \subset C, \lim _{m} \lim _{n}\left|x_{m}+x_{n}\right|=2 \lim _{n}\left|x_{n}\right|$ implies that $\left(x_{n}\right)$ (weakly) converges.

Recall that a $|\cdot|$ norm on a Banach space $X$ is said to be (weakly) locally uniformly rotund if for any $x, x_{n} \in X$ with $|x|=\left|x_{n}\right|=1$ for all $n \in \mathbb{N}$, $\left|x+x_{n}\right| \rightarrow 2$ implies that $x_{n} \rightarrow x$ (weakly).

Note that if $X$ has the $2 R(w 2 R)$-property then $X$ is (weakly) locally uniformly rotund, but the converse is not true [HJ].

The following renorming theorems are due to Odell and Schlumprecht [OS] and Hájek and Johanis [HJ], resp.

Theorem 4.3 ([OS]). Suppose that $X$ is a separable Banach space. Then

(i) there exists an equivalent norm $|\cdot|$ on $X$ such that for any relatively weakly compact sequence $\left(x_{n}\right) \subset X, \lim _{m} \lim _{n}\left|x_{m}+x_{n}\right|=2 \lim _{n}\left|x_{n}\right|$ implies that $\left(x_{n}\right)$ converges;

(ii) therefore, $X$ is reflexive if and only if there is an equivalent norm on $X$ with the $2 R$-property.

Theorem $4.4([\mathrm{HJ}])$. Suppose that $X$ is a Banach space. If there is a continuous one-to-one mapping $T: X \rightarrow c_{0}(\Gamma)$ for some set $\Gamma$, then

(i) there exists an equivalent norm $|\cdot|$ of $X$ such that for any relatively weakly compact sequence $\left(x_{n}\right) \subset X, \lim _{m} \lim _{n}\left|x_{m}+x_{n}\right|=2 \lim _{n}\left|x_{n}\right|$ implies that $\left(x_{n}\right)$ converges weakly; 
(ii) therefore, $X$ is reflexive if and only if there is an equivalent norm on $X$ with the $w 2 R$-property.

By applying Asplund's averaging technique [As] (see also [D1, p. 106]), we can show the following result without any difficulty.

Lemma 4.5. Suppose that $|\cdot|_{1}$ and $|\cdot|_{2}$ are two equivalent norms on a Banach space $X$ such that $|\cdot|_{1}$ has the $2 R(w 2 R)$-property, and the dual of $|\cdot|_{2}$ has the $w 2 R(2 R)$-property. Then there is an equivalent norm $\|\cdot\|$ such that $\|\cdot\|$ has the $2 R(w 2 R)$-property, and the dual of $\|\cdot\|$ has the $w 2 R$ $(2 R)$-property.

THEOREM 4.6. Suppose that $X$ is a closed subspace of a WCG space. Then there is an equivalent $L U R$ norm $|\cdot|$ on $X$ such that for each weakly compact convex set $C \subset X,(C,|\cdot|)$ has the $w 2 R$-property.

Proof. Let $Y$ be a WCG space such that $X \subset Y$. Then, by Troyanski's theorem, there is an equivalent LUR norm \|\|$\cdot \|$ on $Y$. On the other hand, since $Y$ is WCG, there exist a set $\Gamma$ and a bounded linear one-to-one operator $T: Y \rightarrow c_{0}(\Gamma)$ ([AL]; see also [D1]). Therefore, $Y$ can be given an equivalent norm $|\cdot|$ such that for every relatively weakly compact sequence $\left(x_{n}\right) \subset Y$, if

$$
\lim _{m} \lim _{n}\left[2\left(\left|x_{m}\right|^{2}+\left|x_{n}\right|^{2}\right)-\left|x_{m}+x_{n}\right|^{2}\right]=0,
$$

then $\left(x_{n}\right)$ weakly converges. This means that $(C,|\cdot|)$ has the $w 2 R$-property for every weakly compact and convex subset $C$ of $Y$. Finally, let $\|\cdot\|=$ $\||\cdot||+| \cdot \mid$. Then $\|\cdot\|$ is both LUR and $w 2 R$ on each weakly compact and convex subset of $Y$. It is easy to see the restriction of $\|\cdot\|$ to $X$ has the same properties.

Theorem 4.7. Suppose that $C$ is a bounded closed convex and separable subset of a Banach space $X$. Then

(i) $C$ is weakly compact if and only if there exists an equivalent norm $|\cdot|$ on $X$ such that $(C,|\cdot|)$ has the $2 R$-property;

(ii) in particular, if $X$ is separable reflexive then it admits an equivalent $2 R$-norm such that its dual is also $2 R$.

Proof. We need only show (i), since (ii) is just a combination of Theorem 4.3 and Lemma 4.5.

Sufficiency of (i). Suppose that $C$ is a bounded closed convex set in $X$ and $|\cdot|$ is an equivalent norm on $X$ such that $(C,|\cdot|)$ has the $w 2 R$-property. By James' theorem [J], it suffices to show that every functional $x^{*} \in X^{*}$ attains its maximum on $C$. Without loss of generality, we assume that $0 \in C$, and for $x^{*} \in X^{*}$, assume that $\sup _{C} x^{*} \equiv \sup \left\{\left\langle x^{*}, x\right\rangle: x \in C\right\}>0$. (Otherwise, 
we have $\sup _{C} x^{*}=0=\left\langle x^{*}, 0\right\rangle$.) Let $g: \mathbb{R}^{+} \rightarrow \mathbb{R}^{+}$be defined by

$$
g(s)=\sup \left\{\left\langle x^{*}, x\right\rangle: x \in C,|x| \leq s\right\} .
$$

Clearly, $g$ is a continuous function on $\mathbb{R}^{+}$. Let $r_{0}=\min \{s>0: g(s)=$ $\left.\sup _{C} x^{*}\right\}$. Now, we choose $\left(x_{n}\right) \subset C$ with $\left|x_{n}\right| \leq r_{0}$ such that $\lim _{n \rightarrow \infty}\left\langle x^{*}, x_{n}\right\rangle$ $=\sup _{C} x^{*}$. By the definition of $r_{0}$ we have $\left|x_{n}\right| \rightarrow r_{0}$ and

$$
\lim _{m, n \rightarrow \infty}\left|\frac{x_{m}+x_{n}}{2}\right|=r_{0}=\lim _{n}\left|x_{n}\right| .
$$

Therefore, $\left(x_{n}\right)$ is weakly convergent to some element in $C$, say, $x_{0}$. Thus, $\sup _{C} x^{*}=\left\langle x^{*}, x_{0}\right\rangle$, so $x^{*}$ attains its maximum on $C$. Therefore, $C$ is weakly compact.

Necessity of (i). Suppose that $C$ is a separable weakly compact subset of $X \equiv(X,\|\cdot\|)$. Let $X_{0}$ be the closure of $\operatorname{span} C$. Then $X_{0}$ is a separable space and $C$ is again a weakly compact subset of $X_{0}$. By Theorem 4.3, there is an equivalent norm $|\cdot|$ on $X_{0}$ such that for every relatively weakly compact sequence $\left(x_{n}\right) \subset X_{0}$, if $\lim _{m} \lim _{n}\left[2\left(\left|x_{m}\right|^{2}+\left|x_{n}\right|^{2}\right)-\left|x_{m}+x_{n}\right|^{2}\right]=0$, then $\left(x_{n}\right)$ converges. This shows that for every weakly compact convex set $K \subset X_{0}$, $(K,|\cdot|)$ has the $2 R$-property. Now, we finish our proof by extending the norm $|\cdot|$ from $X_{0}$ to the whole space $X$ to be an equivalent norm of $X$. Indeed, this can be done by the following procedure. Choose any $N>L$ (where $L$ denotes the Lipschitz constant of the norm $|\cdot|$ on $\left.X_{0}\right)$. For $x \in X$ we define

$$
\|x\|_{N}=\inf \left\{|y|+N\|x-y\|: y \in X_{0}\right\} .
$$

Then $\|\cdot\|_{N}$ is an equivalent norm on $X$ with the Lipschitz norm at most $N$ (see, for instance, [WC2] or [WCHL]) such that its restriction to $X_{0}$ is just $|\cdot|$.

TheOrem 4.8. Suppose that $C$ is a bounded closed convex subset of a Banach space $X$. Then

(i) $C$ is weakly compact if and only if there exists an equivalent norm $|\cdot|$ on $X$ such that $(C,|\cdot|)$ has the w2R-property;

(ii) $X$ is reflexive if and only if $X$ admits an equivalent $w 2 R$-norm whose dual norm is also $w 2 R$.

Proof. Sufficiency of (i). The sufficiency is completely contained in the proof of "Sufficiency of (i)" of Theorem 4.7.

Necessity of (i). Suppose that $C$ is a weakly compact subset of $X \equiv$ $(X,\|\cdot\|)$. Let $X_{0}$ be the closure of $\operatorname{span} C$. Then $X_{0}$ is a WCG space and $C$ is again a weakly compact subset of $X_{0}$. By Theorem 4.4, there is an equivalent norm $|\cdot|$ on $X_{0}$ such that for every relatively weakly compact sequence $\left(x_{n}\right) \subset X_{0}$, if $2\left(\left|x_{m}\right|^{2}+\left|x_{n}\right|^{2}\right)-\left|x_{m}+x_{n}\right|^{2} \rightarrow 0$, then $\left(x_{n}\right)$ converges weakly. This implies that for every weakly compact convex set $K \subset X_{0}$, 
$(K,|\cdot|)$ has the $w 2 R$-property. Now, we finish our proof by extending the norm $|\cdot|$ again from $X_{0}$ to $X$ to be an equivalent norm of $X$.

(ii) It is just a direct consequence of Theorem 4.4 and Lemma 4.5.

Corollary 4.9. A Banach space is WCG if and only if there exist a closed convex fundamental set $C \subset X$ and an equivalent norm that is $w 2 R$ on $C$.

Acknowledgements. The authors would like to thank the referee for his helpful comments, suggestions and careful corrections on the previous version of this manuscript. They are very grateful to Professor Tadeusz Figiel for his friendly and helpful conversations and corrections to this paper.

Research of L. X. Cheng was supported by the NSFC grants: 10771175 and 11071201. Research of Q. J. Cheng was supported by the NSFC.

\section{References}

[AL] D. Amir and J. Lindenstrauss, The structure of weakly compact sets in Banach spaces, Ann. of Math. 88 (1968), 35-46.

[As] E. Asplund, Averaged norms, Israel J. Math. 5 (1967), 227-233.

[CCLZ] L. X. Cheng, Q. J. Cheng, Z. H. Luo and W. Zhang, Every weakly compact set can be uniformly embedded into a reflexive space, Acta Math. Sinica (Engl. Ser.) 25 (2009), 1109-1112.

[CLS] L. X. Cheng, S. Z. Shi and E. S. Lee, Generic Fréchet differentiability of convex functions on non-Asplund spaces, J. Math. Anal. Appl. 214 (1997), 367-377.

[CW] L. X. Cheng and C. X. Wu, The $w^{*}$-Fréchet differentiability property, the RadonNikodym property and $w^{*}$-Asplund spaces, Acta Math. Sinica (Chin. Ser.) 46 (2003), 385-390 (in Chinese).

[CZ] L. X. Cheng and W. Zhang, A note on nonsupport points, negligible sets, Gâteaux differentiability and Lipschitz embeddings, J. Math. Anal. Appl. 350 (2009), 531-536.

[DFJP] W. J. Davis, T. Figiel, W. B. Johnson and A. Pełczyński, Factoring weakly compact operators, J. Funct. Anal. 17 (1974), 311-327.

[DGZ] R. Deville, G. Godefroy and V. Zizler, Smoothness and Renormings in Banach Spaces, Pitman Monogr. Surveys Pure Appl. Math. 64, Longman Sci. \& Tech. and Wiley, New York, 1993.

[D1] J. Diestel, Geometry of Banach Spaces, Lecture Notes in Math. 485, Springer, 1975.

[D2] - Sequences and Series in Banach Spaces, Springer, New York, 1984.

[FGMZ] M. Fabian, G. Godefroy, V. Montesinos and V. Zizler, Inner characterizations of weakly compactly generated Banach spaces and their relatives, J. Math. Anal. Appl. 297 (2004), 419-455.

[FMZ1] M. Fabian, V. Montesinos and V. Zizler, Weakly compact sets and smooth norms in Banach spaces, Bull. Austral. Math. Soc. 65 (2002), 223-230.

[FMZ2] -, 一, 一, Weak compactness and $\sigma$-Asplund generated Banach spaces, Studia Math. 181 (2007), 125-152.

[Fl] K. Floret, Weakly Compact Sets, Lecture Notes in Math. 801, Springer, 1980. 
[HJ] P. Hájek and M. Johanis, Characterization of reflexivity by equivalent renorming, J. Funct. Anal. 211 (2004), 163-172.

[J] R. C. James, Reflexivity and the sup of linear functionals, Israel J. Math. 13 (1972), 289-300.

[L] J. Lindenstrauss, Weakly compact sets-their topological properties and the Banach spaces they generate, in: Symposium on Infinite-Dimensional Topology (Baton Rouge, LA, 1967), Ann. of Math. Stud. 69, Princeton Univ. Press, 1972, 235-273.

[OS] E. Odell and T. Schlumprecht, Asymptotic properties of Banach spaces under renormings, J. Amer. Math. Soc. 11 (1998), 175-188.

[P] R. R. Phelps, Convex Functions, Monotone Operators and Differentiability, Lecture Notes in Math. 1364, Springer, 1989; 2nd ed., 1993.

[T] S. Troyanski, On locally uniformly convex and differentiable norms in certain nonseparable Banach spaces, Studia Math. 43 (1972), 125-138.

[WC1] C. X. Wu and L. X. Cheng, Extensions of the Preiss differentiability theorem, J. Funct. Anal. 124 (1994), 112-118.

[WC2] - - - A note on the differentiability of convex functions, Proc. Amer. Math. Soc. 121 (1994), 1057-1062.

[WCHL] C. X. Wu, L. X. Cheng, M. H. Ha and E. S. Lee, Convexification of nonconvex functions and application to minimum and maximum principles for nonconvex sets, Comput. Math. Appl. 31 (1996), 27-36.

Lixin Cheng, Qingjin Cheng, Zhenghua Luo

School of Mathematical Sciences, Xiamen University

Xiamen, 361005, China

E-mail: lxcheng@xmu.edu.cn qjcheng@xmu.edu.cn

luozhenghua@hotmail.com

Received October 7, 2009

Revised version September 23, 2010 